\title{
HIV exposure: Counselling guidelines
}

The Canadian Medical Association (CMA) has produced a booklet of counselling guidelines for human immunodeficiency virus (HIV) antibody testing. The booklet covers such topics as serologic tests for HIV infection, prerequisites for testing, pre- and post test procedures, occupational exposure, and CMA policies on HIV/AIDS. The following excerpts are published with permission from the Canadian Medical Association. Human Immunodeficiency Virus Antibody Testing. Ottawa: Canadian Medical Association, 1990:5.9.

\section{Minimum information to give patients about HIV and AIDS}

- HIV infection can produce a variety of illnesses including enlarged lymph glands, gastrointestinal symptoms and wasting, neurological syndromes and immunodeficiency, but most infected people remain asymptomatic and appear healthy for many years after HIV infection begins, and some people may never develop illness because of HIV infection.

- Once a person is infected with HIV, and regardless of whether or not the person has developed any illness, that person is infectious for life and capable of transmitting HIV to other persons by specific activities.

- HIV is found in blood, semen, genital fluids, and breast milk and, when a person is infected, depositing any of them in someone else's body can infect that person.

- Activities which can transmit HIV include sexual intercourse; sharing of injection equipment during nonmedical injection drug use; donation of blood, tissue, sperm or ova; conception in an infected woman; and possibly breast feeding. Other activities have never been shown convincingly to transmit HIV.

- Being exposed to HIV through these activities does not necessarily mean that the exposed person has been infected. The only effective way to determine if a person who has been exposed to HIV is infected is to undergo HIV antibody testing.

- Testing cannot determine if someone has been infected until sufficient time has elapsed for antibodies to be produced, and this can be as long as six months.

\section{Occupational exposure to HIV}

Occupationally acquired HIV infection of health care workers is a rare event. Except for three workers splashed with infected blood, transmission has only occurred from accidental percutaneous exposure. Body fluids other than blood have never been convincingly shown to transmit HIV. The risk of occupationally acquired HIV infection has been estimated to be $0.4 \%$ of parenteral injuries.

Knowing that an injury has exposed a health care worker to HIV is a very distressing situation for the worker. Not knowing if the patient who is the source of the exposure is HIV infected compounds this stress. There is intense pressure to learn if the patient is or is not infected, especially if the exposed worker wants to receive a short course of AZT prophylaxis in an attempt to prevent HIV infection, although the efficacy of this prophylaxis is unproven.

When it is not known if the patient is infected, then the patient should be asked to undergo testing. As with testing any other patient, informed consent and pre- and post test counselling are essential. Further, the patient should be asked to consent to communication of test results to the exposed worker, so that the worker can receive appropriate counselling, education, support and care. While most patients are likely to consent to such testing, some may refuse. The general rule is that such refusals must be respected. As an exception, in some unusual circumstances, compulsory testing may be ethically and legally justified - for example, when the exposed worker wants to begin AZT prophylaxis but cannot do so unless the patient is HIV antibody seropositive.

- Decreasing the number of partners may reduce but cannot eliminate the risk of exposure; only eliminating risk-producing activities will do this.

- Exposure to HIV can be avoided by abstinence, by mutual monogamy with an uninfected sexual partner, and by not sharing injection equipment during nonmedical drug use; the risk of HIV infection can be reduced by the proper use of latex condoms or other barriers during sexual intercourse and by disinfecting shared injection equipment between uses. $\square$ 


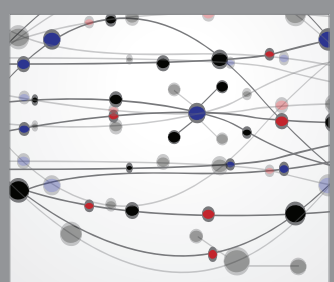

The Scientific World Journal
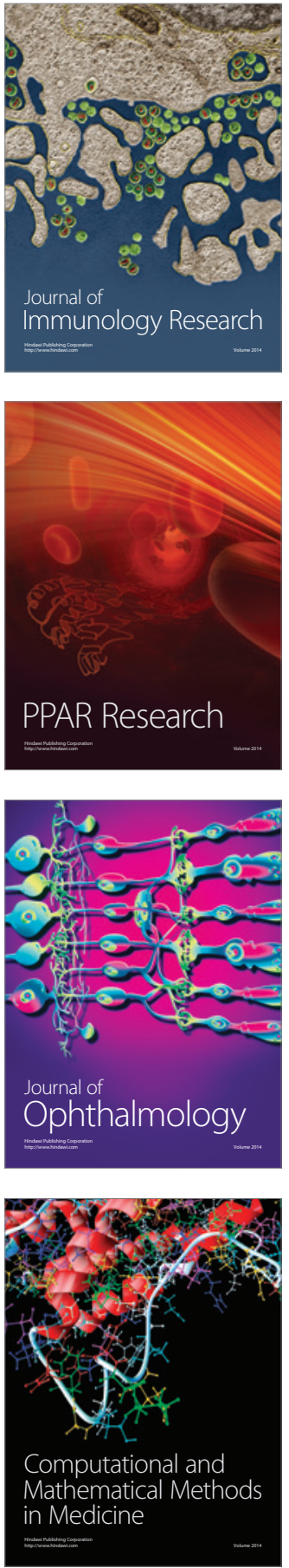

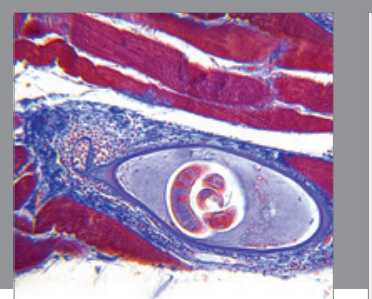

Gastroenterology Research and Practice

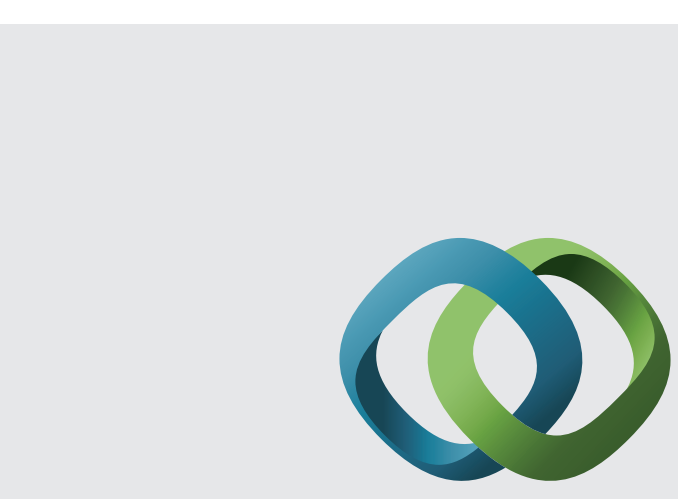

\section{Hindawi}

Submit your manuscripts at

http://www.hindawi.com
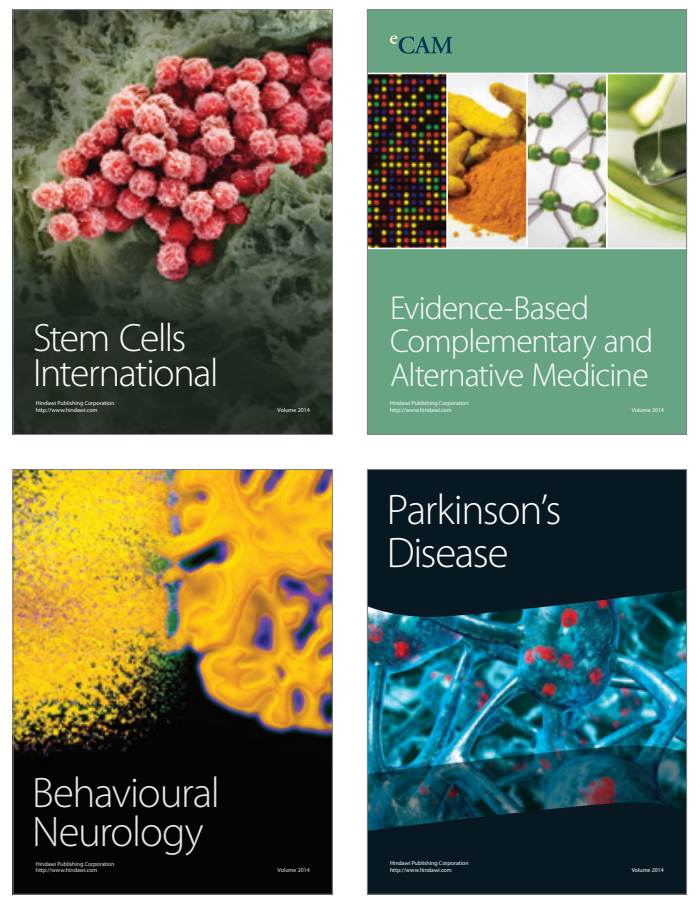
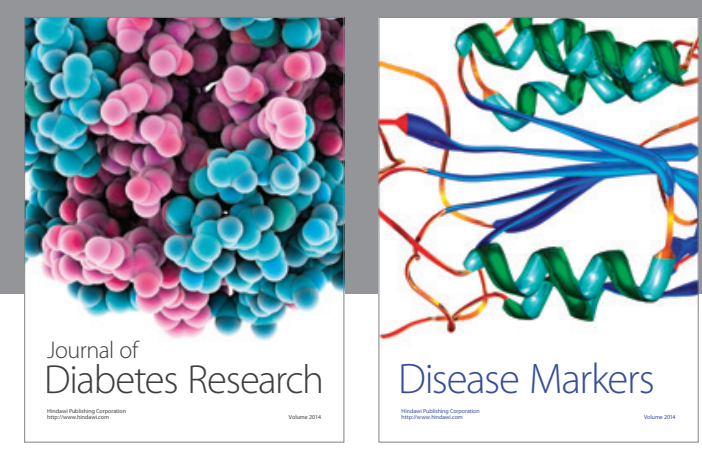

Disease Markers
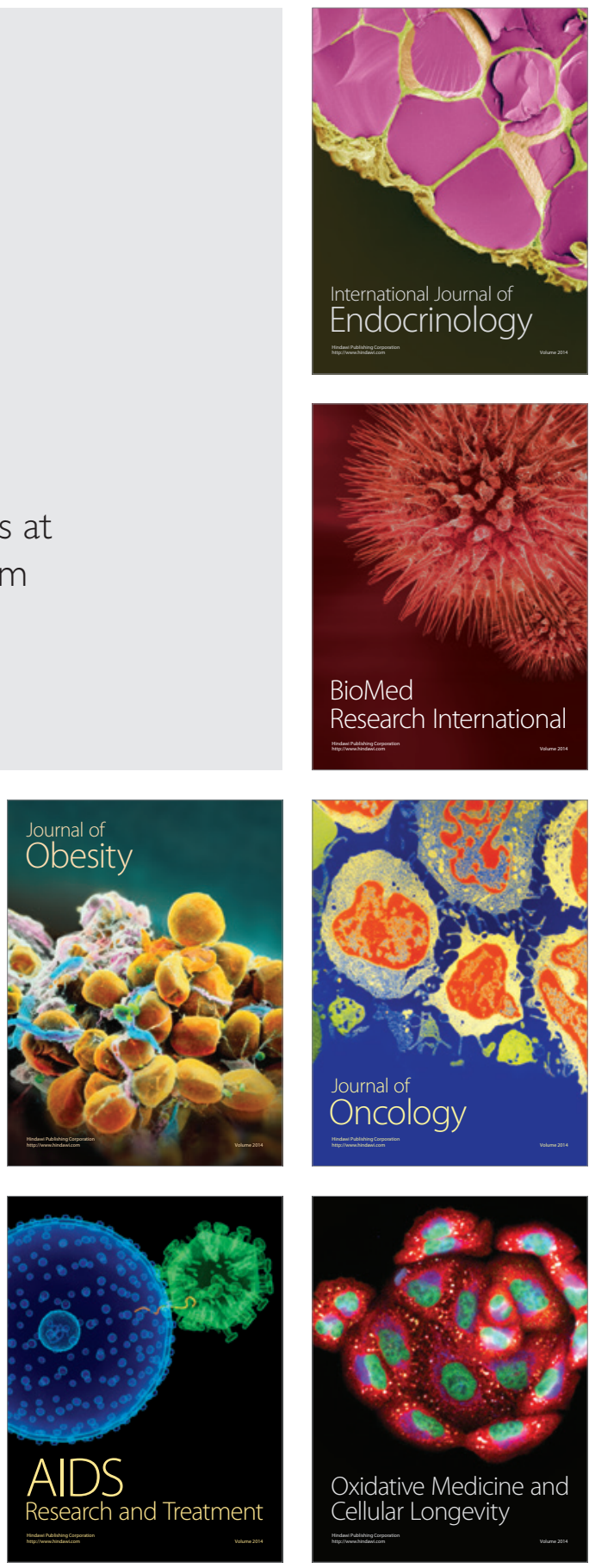